Missouri Orthopaedic Institute University of Missouri

\section{Does Gastric Bypass Surgery Increase the risk of Complications for Fracture ORIF?}

Conor A. Smith, BS, Brett D. Crist, MD
14 Orthopaedic Surgery

University of Missouri Health

\section{Introduction}

Roux-en-y Gastric Bypass (RYGB) surgery is the most used method of bariatric surgery techniques historically and currently (around $50 \%$ of all), with over 200,000 procedures performed in the US alone, and it provides consistent results. ${ }^{1}$ However, continued compliance with diet supplementation post-surgery tends to decline and the concern is that patients may become malnourished. As a result of poor compliance and nutritional malabsorption, there is an increased risk of fracture.

- $96 \%$ of pre-bariatric surgery patients are Vitamin D deficient

- Studies show loss of bone mineral density

- Axelsson reviewed 33,000 RYGB patients and found that fracture risk is higher after first year for all fracture types

- These are consistent with fragility type fractures (hips, distal radius, etc.)

The purpose of this study was to evaluate the risk of complication after open reduction and internal fixation (ORIF) fracture in these patients.

Hypothesis: Due to the declining nutritional status after RYGB, the risk of complication (infection, nonunion, etc.) is higher in these patients after ORIF.

\section{Methods}

After IRB approval, 30 patients were identified who had previous gastric bypass surgery and subsequently had fracture ORIF. A retrospective chart review including date of gastric bypass, body mass index (BMI) at time of gastric bypass, type of fracture and mechanism of injury (high vs low energy), date of fracture, BMI at time of fracture, and presence of comorbidities-nicotine and non-steroidal anti-inflammatory drug use and immunotherapy. Primary endpoints any unplanned surgery related to their fracture-such as infection or nonunion.

\section{Results}

Patients average age at the time of fracture was 49 years with an average $\mathrm{BMI}$ of $30.66 \mathrm{~kg} / \mathrm{m}^{2}$. At the time of fracture, the average BMI decrease after gastric bypass was $15.1 \mathrm{~kg} / \mathrm{m}^{2}$. The average time between gastric bypass and fracture was 1461 days (4 years). Type II Diabetes was noted in $33.3 \%(10 / 30)$ patients. Fractures sustained included distal radius fractures $30 \%$ (9/30), and ulnar, tibia/fibula, and femur fractures at $13.33 \%(4 / 30)$ each. Fall from standing was the most common mechanism of injury in $55.2 \%(16 / 30)$, followed by MVC at $27.6 \%(8 / 30)$. Seven (23\%) patients experienced complications requiring operative management including non-union $(4 / 30,13.3 \%)$, secondary fractures $(2 / 30,6.67 \%)$, and deep infection $(1 / 30,3.3 \%)$

1461 Average Time (Days) elapsed between RYGB and fracture 1461

30.66 Average BMI at time of fracture

1

15.1 Drop in BMI before fracture

1

33.3\% Patients with concomitant Diabetes Type II

1

$30 \%$ Distal radius fractures $(9 / 30)$

1

$55.2 \%$ Fall from less than standing height $(16 / 30)$

\section{Discussion}

Anecdotal experience roused suspicion for higher complication rates in this population. Patients were on average less than 50 years old, though their injuries were more consistent with osteoporotic patients - i.e. mechanism of injury and distal radius fractures. Consistent with large cohorts of gastric bypass patients who had increased fracture risk after 1 year postRYGB, our patient group did not show any fractures within 1 year of surgery. Nonunion rates among this cohort were higher. In addition, site specific nonunion rates were higher than average for those that experienced nonunion.

Limitations include the retrospective review and the small cohort. Larger prospective cohorts should be investigated to gain true incidence of complications. All of the complication rates in our cohort exceeded those expected for non-gastric bypass patients-including infection, nonunion, and secondary fractures.

\section{Conclusion}

This preliminary study showed that patient status post gastric bypass have a high complication rate associated with fracture ORIF. 